\title{
Thermal conductivity studies on magnetite nanofluids coated with short-chain and long-chain fatty acid surfactants
}

\author{
R LENIN, A DADWAL and P A JOY* \\ Physical and Materials Chemistry Division, Academy of Scientific and Innovative Research (AcSIR), CSIR-National \\ Chemical Laboratory, Pune 411008, India \\ *Author for correspondence (pa.joy@ncl.res.in)
}

MS received 20 March 2018; accepted 21 May 2018; published online 31 August 2018

\begin{abstract}
The effect of the length of surfactant molecules on the surface of the nanoparticles on the thermal conductivity of nanofluids is studied. Magnetite $\left(\mathrm{Fe}_{3} \mathrm{O}_{4}\right)$ nanoparticles of comparable sizes are stabilized with short-chain capric acid $\left(\mathrm{C}_{9} \mathrm{H}_{19} \mathrm{COOH}\right)$ and long-chain stearic acid $\left(\mathrm{C}_{17} \mathrm{H}_{35} \mathrm{COOH}\right)$ molecules. Thermal conductivity of the two surfactant-coated magnetite nanoparticles dispersed in toluene is measured as a function of the concentration of the particles in the fluids and in the presence of a magnetic field. Studies showed that the critical concentration for thermal conductivity enhancement is lower for stearic-acid-coated fluid as compared with the capric-acid-coated fluid. Comparable enhancement in the thermal conductivity is observed at higher concentrations of the particles. Relatively larger enhancement in the thermal conductivity is observed for the capric-acid-coated fluid in a magnetic field. The difference in the enhancement in the thermal conductivity, depending on the chain length of the surfactant, is explained in terms of the inter-particle magnetic interactions and formation of clusters.
\end{abstract}

Keywords. Magnetic nanofluids; surfactant; capric acid; stearic acid; thermal conductivity; magnetic field.

\section{Introduction}

The surfactant coated on the surface of nanoparticles is known to play an important role in determining the dispersion and stability of nanofluids [1,2]. The chemistry of the surface of the surfactant-coated nanoparticles is decided by the choice of the surfactants and the nature of stabilization. Fatty acids are one of the major surfactants studied for the stabilization of magnetite nanoparticles dispersed in aqueous as well as non-aqueous medium [3-7]. The surfactant layer on the surface of the nanoparticles not only stabilizes the nanoparticles in the carrier liquid but also makes changes in the physical, chemical and thermophysical properties of the nanofluids $[1,8-12]$. The effective thickness of the surfactant layer determines the hydrodynamic diameter of the nanoparticles in a fluid and the inter-particle distance between the nanoparticle [13]. Therefore, the amount of surfactant on the surface of a nanoparticle and the length of the surfactant molecules also influence the thermophysical properties of the nanofluids.

The amount of the non-magnetic organic surfactant layer on the surface of a magnetic nanoparticle is known to change the magnetic properties of the nanoparticles. Fu et al [14] observed a decrease in the magnetization of lauric acid (dodecanoic acid)-coated $\mathrm{Fe}_{3} \mathrm{O}_{4}$ nanoparticles compared with that of uncoated $\mathrm{Fe}_{3} \mathrm{O}_{4}$ nanoparticles. The authors also observed a further decrease in the magnetization by coating with capric acid as a secondary surfactant layer over the lauric acid (primary layer)-coated nanoparticles. Barbeta et al [15] studied the magnetic properties of $\mathrm{Fe}_{3} \mathrm{O}_{4}$ nanoparticles coated with lauric acid and oleic acid as surfactants. The authors observed an increase in the superparamagnetic blocking temperature for the nanofluid with increasing concentration of the nanoparticles in the fluid due to the increased inter-particle interactions. However, the authors did not observe any appreciable change in the overall magnetic behaviour by changing the surfactant on the surface of the nanoparticles.

The hydrodynamic diameter of the nanoparticles in a fluid depends on the amount and thickness of the surfactant layer on the surface of the nanoparticles. The Brownian relaxation of the particles (particle rotation) mainly depends on the hydrodynamic diameter. Regmi et al [16] prepared $\mathrm{Fe}_{3} \mathrm{O}_{4}$ nanoparticles of comparable size $(12 \mathrm{~nm})$ and coated with fatty acid surfactants of different chain lengths (lauric, myristic and oleic acid) and studied the hydrodynamic contribution (Brownian relaxation) for hyperthermia and magneto-optic characteristics by keeping the Neel's contribution to relaxation as constant. From the studies, it was found that the magnetohydrodynamic behaviour of the magnetic fluids can be controlled by choosing the appropriate surfactant. Vekas et al [17] studied the rheological behaviour of oleic-acidcoated magnetite nanoparticles dispersed in non-polar (transformer oil) and polar (pentanol, heptanol and dioctylsebacate) solvents and they found that the rheological behaviour of magnetic fluids is highly sensitive to the type of stabilization 
(monolayer/bilayer or hydrophobic/hydrophilic) and quality of the surfactants.

The thickness of the surfactant layer also influences the size and distribution of the stabilized nanoparticles. Vekas et al [13] used fatty acid surfactants of different chain lengths to stabilize magnetite nanoparticles and it was found that short-chain-length surfactants (lauric and myristic acids) stabilize smaller particles and longer-chain-length surfactant (oleic acid) stabilizes a wide range of particle sizes. The nanoparticles stabilized by short-chain surfactant showed narrow size distributions, whereas longer-chain-length surfactant gave a broader distribution. The authors also observed different initial susceptibilities and different magneto-viscous effects for the magnetite nanoparticles coated with surfactants of different chain lengths. The initial susceptibility and relative viscosity in the presence of a magnetic field are found to be less for the short-chain-stabilized nanoparticles than the oleic-acid-stabilized magnetite nanoparticles dispersed in transformer oil. Avdeev et al [18] studied saturated fatty acid surfactants with different chain lengths (lauric, myristic, palmitic and stearic acids) for the stabilization of magnetite nanoparticles in a non-polar solvent (decahydronaphthalene) and compared the results to those for oleic-acid-coated magnetite nanoparticles. From the results, the authors found that all the saturated fatty acid surfactants showed similar stabilizing properties and similar size distribution, whereas the oleic-acid-coated nanoparticles showed broader size distribution. Avdeev et al [19] also studied shortchain fatty acids for stabilization of magnetite nanoparticles and found that the chain length is an important parameter that determines the organization of the surfactant molecules on the surface of the nanoparticles and that the short-chain surfactants stabilize smaller particles with reduced polydispersity. The effect of the surfactant on the heat transfer properties, using thiolate-coated Au nanoparticles dispersed in toluene and citrate-coated Au nanoparticles dispersed in water, has been reported by Patel et al [20]. It was found that the thiolate-coated $\mathrm{Au}$ nanoparticles are less effective in thermal conductivity enhancement as compared with the citrate-coated Au nanoparticles. From the results, it was concluded that the effective heat transfer at the surface of the nanoparticles is decided by the type of surfactant coating.

From these discussions, it is clear that the thickness of the surfactant layer (related to physical length of the surfactant molecule) on the surface of the nanoparticle is one of the important factors to be considered for studying the thermophysical properties of the nanofluids. So far there are no reports on the effect of surfactant layer thickness on the thermal conductivity of nanofluids. In the present study, we have compared the thermal conductivity of the magnetite nanoparticles coated with short-chain fatty acid, capric acid (decanoic acid), and the long-chain fatty acid, stearic acid (octadecanoic acid), synthesized under identical conditions and with comparable particle size. The changes in the thermal conductivity of the magnetite nanofluids containing the fatty-acid-coated magnetite nanoparticles for different concentrations as well as for different strengths of applied magnetic field are studied.

\section{Experimental}

\subsection{Materials}

For the synthesis of fatty-acid-coated nanoparticles, ferric chloride hexahydrate $\left(\mathrm{FeCl}_{3} \cdot 6 \mathrm{H}_{2} \mathrm{O}, 97 \%\right)$, ferrous chloride tetrahydrate $\left(\mathrm{FeCl}_{2} \cdot 4 \mathrm{H}_{2} \mathrm{O}, 99 \%\right)$, capric acid (decanoic acid, 98\%) and stearic acid (octadecanoic acid, 95\%) were purchased from Sigma-Aldrich Chemicals. Aqueous ammonia $\left(\mathrm{NH}_{4} \mathrm{OH}, 25 \%\right)$, hydrochloric acid $(37 \%)$, acetone $(99.5 \%)$, hexane $(95 \%)$, iso-propanol $(99.5 \%)$ and toluene $(99.5 \%)$ were purchased from Merck chemicals, India. All the chemicals were used as-received without any further purification and double-distilled water was used throughout the synthesis procedure.

\subsection{Synthesis}

The magnetite nanoparticles coated with short-chain capric acid and the long-chain stearic acid were synthesized by the co-precipitation method using the iron chloride precursors $\left(\mathrm{FeCl}_{3} \cdot 6 \mathrm{H}_{2} \mathrm{O}\right.$ and $\left.\mathrm{FeCl}_{2} \cdot 4 \mathrm{H}_{2} \mathrm{O}\right)$ and ammonium hydroxide $\left(\mathrm{NH}_{4} \mathrm{OH}\right)$ as the precipitant, in the presence of the corresponding surfactant in the reaction medium. Capric acid dissolved in $20 \mathrm{ml}$ acetone (for capric acid coating) and stearic acid dissolved in $20 \mathrm{ml}$ of 2-propanol (for stearic acid coating) were used. Details of the synthesis are reported previously $[21,22]$. After the precipitation reaction, a dispersion obtained was washed with 1:1 acetone-hexane mixture (in the case of capric acid) and 1:1 2-propanol-hexane mixture (in the case of stearic acid). The nanoparticles in the aqueous layer were transferred to the non-aqueous layer (hexane) during the washing process and the non-aqueous layer containing the nanoparticles was separated using a separating funnel and dried at room temperature to obtain nanoparticles in the powder form. The dried powder samples were labelled as MCA and MST, respectively, for the capric-acid- and stearic-acidcoated samples, and used for further studies. Nanofluids were prepared by dispersing the dried nanoparticles in toluene at different concentrations (volume \%) and used for thermal conductivity studies.

\subsection{Characterization techniques}

Powder X-ray diffraction (XRD) studies on the powder samples were carried out on a PANalytical X' pert Pro diffractometer using $\mathrm{Cu} \mathrm{K} \alpha(1.5418 \AA)$ radiation. Morphology of the powders was studied using a transmission electron microscope (TEM) FEI model TECNAI G2 F20. Infrared spectra (IR) of the powder samples were recorded on a PerkinElmer Spectrum-one FT-IR spectrometer by making pellets with $\mathrm{KBr}$. Thermogravimetric analysis (TGA) of the dried 
powder sample was performed on an SDT Q600 TG-DTA analyser in nitrogen atmosphere. Magnetic measurements were performed on a SQUID-VSM (Quantum Design). The thermal conductivity of the fluids was measured using a homemade cell based on the principle of Transient Hot Wire (THW) method, where a platinum wire was used as the heat source [21]. The uncertainty in the thermal conductivity measurement was $\pm 1 \%$. Thermal conductivity measurement in the presence of a DC magnetic field parallel to the temperature gradient was performed up to $1 \mathrm{~T}$ produced by an electromagnet.

\section{Results}

\subsection{Characterization of powder samples}

The XRD patterns of the as-synthesized powder samples, MCA and MST, are compared to the simulated pattern of $\mathrm{Fe}_{3} \mathrm{O}_{4}$ (magnetite) in figure 1 . The positions and intensities of all the peaks in the XRD patterns of the two samples match very well with the simulated pattern, showing the formation of magnetite. The cubic lattice parameter is calculated as 8.39 and $8.40 \AA$ for MCA and MST, respectively, which is comparable to that of magnetite (8.40 $\AA$, JCPDS \#19-0629). The average crystallite size is calculated from the full-width at half-maximum (FWHM) of the major peak (311) using the Scherrer equation as $6 \pm 1$ and $7 \pm 1 \mathrm{~nm}$ for MCA and MST, respectively.

TEM images of the capric-acid- and stearic-acid-coated samples and their corresponding particle size histogram are shown in figure 2. The average particle sizes obtained from the Gaussian fitted particle size histogram are 7.4 and $9.1 \mathrm{~nm}$ for MCA and MST, respectively. For both samples, the average particle size is slightly larger than the average crystallite

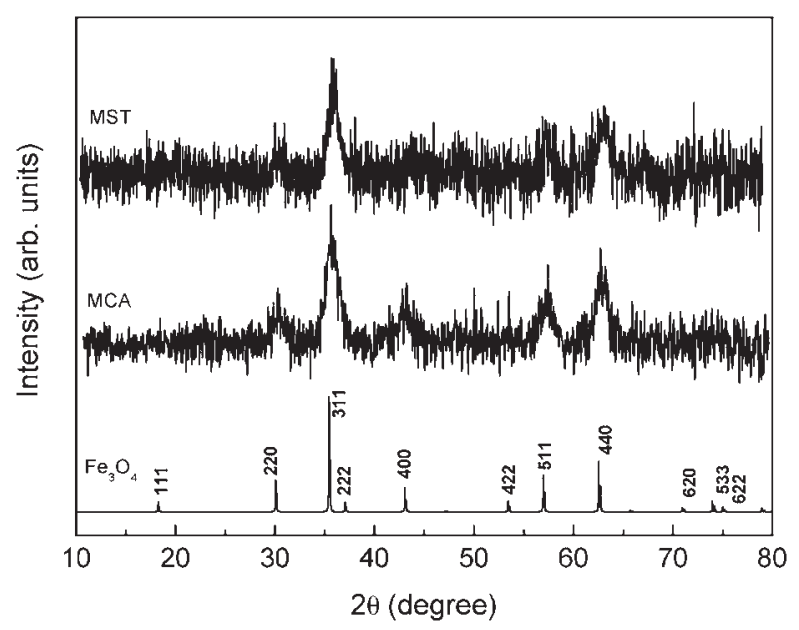

Figure 1. Powder XRD patterns of capric acid (MCA) and stearic acid (MST)-coated magnetite nanoparticles. The simulated pattern of $\mathrm{Fe}_{3} \mathrm{O}_{4}$ is shown and indexed at the bottom for comparison. size calculated from the XRD patterns using the Scherrer equation. The larger size obtained from the TEM images could be due to the amorphous layer and the surfactant layer present on the surface of the nanoparticles. The chain length of the fatty acid attached to the surface of the nanoparticles may contribute towards the observed larger size of the nanoparticles. For example, the length of capric acid and stearic acid molecules are reported as 1.4 and $2.4 \mathrm{~nm}$, respectively [23-26]. The observed size distribution is slightly larger for the stearic-acid-coated sample as compared with the capricacid-coated sample and this could be due to the fact that surfactant with larger carbon chain length stabilizes nanoparticles in a wider size range and short chain fatty acid stabilizes only smaller particles, as reported [13]. The stearic-acidcoated sample (MST) shows highly aggregated particles in the TEM image, but the capric-acid-coated sample (MCA) shows well-separated particles with small clusters (figure 2). However, in both cases, the individual particles are separated from one another due to the surface coating with the fatty acid molecules.

Thermogravimetric analysis curves of capric- and stearic-acid-coated samples are shown in figure 3. The total weight losses below $500^{\circ} \mathrm{C}$ are obtained as $26 \%$ and $18 \%$, respectively, for MCA and MST. Both samples show threestep weight loss below $500^{\circ} \mathrm{C}$. The first weight loss below $150^{\circ} \mathrm{C}$ corresponds to the loss of solvent molecules and/or moisture, and the second and third weight losses correspond to the removal of the surfactant molecules. The second weight loss for both the samples is due to the loss of weakly bound secondary surfactant layer over the primary layer and the third weight loss corresponds to removal of the primary layer of the surfactants $[6,22,27]$. Thus, the thermogravimetric analysis of the samples MCA and MST indicates that there are two groups of surfactants attached to the nanoparticle surfaces. The capric- and stearic-acid-coated samples show almost the same amount of the primary surfactant. However, the amount of the secondary surfactant on the surface of the nanoparticles is more for the capric-acid-coated sample compared with the stearic-acid-coated sample (table 1).

To obtain information on the nature of bonding of the surfactant molecules with the surface of the nanoparticles, IR spectroscopy studies have been carried out. Figure 4 shows a comparison of the IR spectra of the fatty-acid-coated samples to that of the corresponding neat fatty acids. The bands at 2850 and $2920 \mathrm{~cm}^{-1}$ in the spectra of neat acids and the coated samples correspond, respectively, to symmetric and asymmetric $\mathrm{C}-\mathrm{H}$ stretching vibrations of the methylene $\left(-\mathrm{CH}_{2}-\right)$ groups. The strong band at $1708 \mathrm{~cm}^{-1}$ in the spectra of capric acid and at $1702 \mathrm{~cm}^{-1}$ for stearic acid corresponds to the carbonyl stretching vibration of the carboxylic group. The intensity of this band is reduced to a large extent in the spectra of coated nanoparticles. The bands at 930 and $942 \mathrm{~cm}^{-1}$ in the spectra of CA and ST, respectively, are characteristic bands for dimeric form of fatty acid molecules, which correspond to $\mathrm{O}-\mathrm{H}$ bending vibration. This band is completely vanished in the spectra of MCA and MST. The new band over 


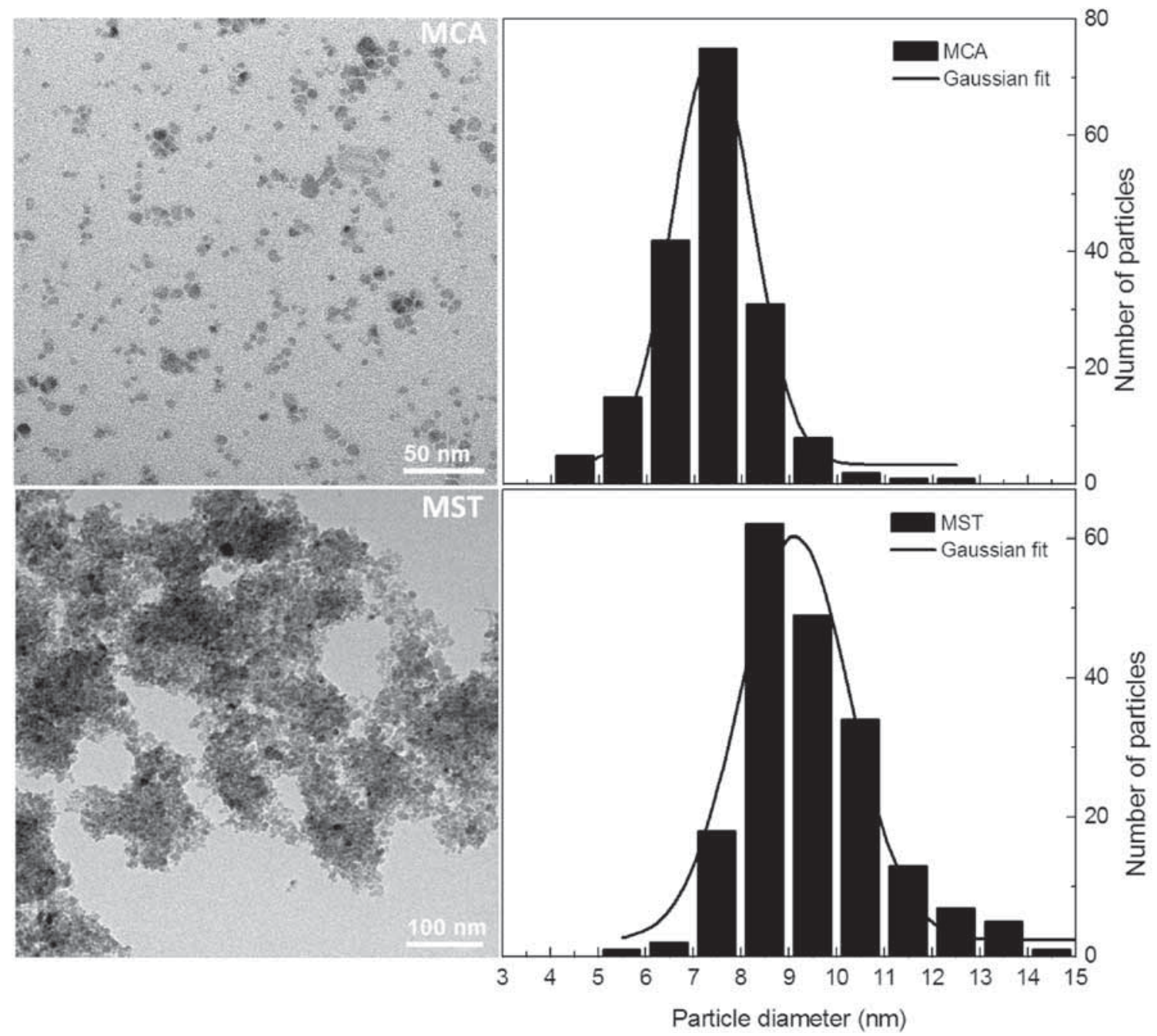

Figure 2. TEM images (left) and the corresponding particle size histogram (right) of the capric acid (MCA) and stearic acid (MST)-coated samples.

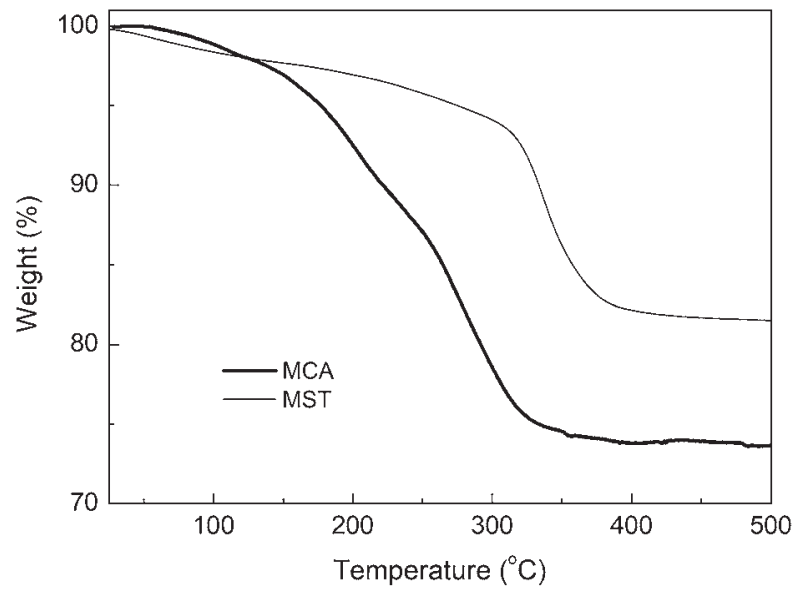

Figure 3. Thermogravimetric analysis curves of capric acid (thick line) and stearic acid (thin line)-coated samples.
610-620 $\mathrm{cm}^{-1}$ in the spectra of both the coated samples corresponds to $\mathrm{Fe}-\mathrm{O}$ stretching frequency of $\mathrm{Fe}_{3} \mathrm{O}_{4}$, including the $\mathrm{Fe}-\mathrm{O}$ bond due to the attachment of the fatty acid to the $\mathrm{Fe}$ atom on the surface of the nanoparticles through the oxygen atoms of the carboxyl group [28].

A new band appears in the spectra of the capric-acid-coated sample at 1536 and at $1523 \mathrm{~cm}^{-1}$ in the spectra of the stearicacid-coated sample. This band corresponds to the asymmetric stretching vibration of the carboxylate group $\left(-\mathrm{COO}_{\text {asym }}^{-}\right)$ [29]. Detailed analysis of the IR spectra in the carboxylate vibrational region suggested chelating bidentate coordination of the carboxylate group to the iron atom on the surface of the nanoparticles, as reported $[22,29,30]$. The weak band over $1700-1710 \mathrm{~cm}^{-1}$ in the spectra of both the coated samples is due to the secondary surfactants [31]. Intensity of this band is relatively larger for MCA than for MST, corresponding to relatively larger amount of secondary 
Table 1. Amount of total, primary and secondary surfactants on the capric- and stearic-acid-coated magnetite nanoparticles, and the relative thermal conductivity of the corresponding nanofluids at 4 vol\%.

\begin{tabular}{lcccc}
\hline Sample code & Total surfactant $( \pm 1 \%)$ & Primary surfactant $( \pm 1 \%)$ & Secondary surfactant $( \pm 1 \%)$ & $k / k_{\mathrm{f}} @ 4$ vol\% \\
\hline MCA & 26 & 13 & 13 & 1.09 \\
MST & 18 & 12 & 6 & 1.11 \\
\hline
\end{tabular}
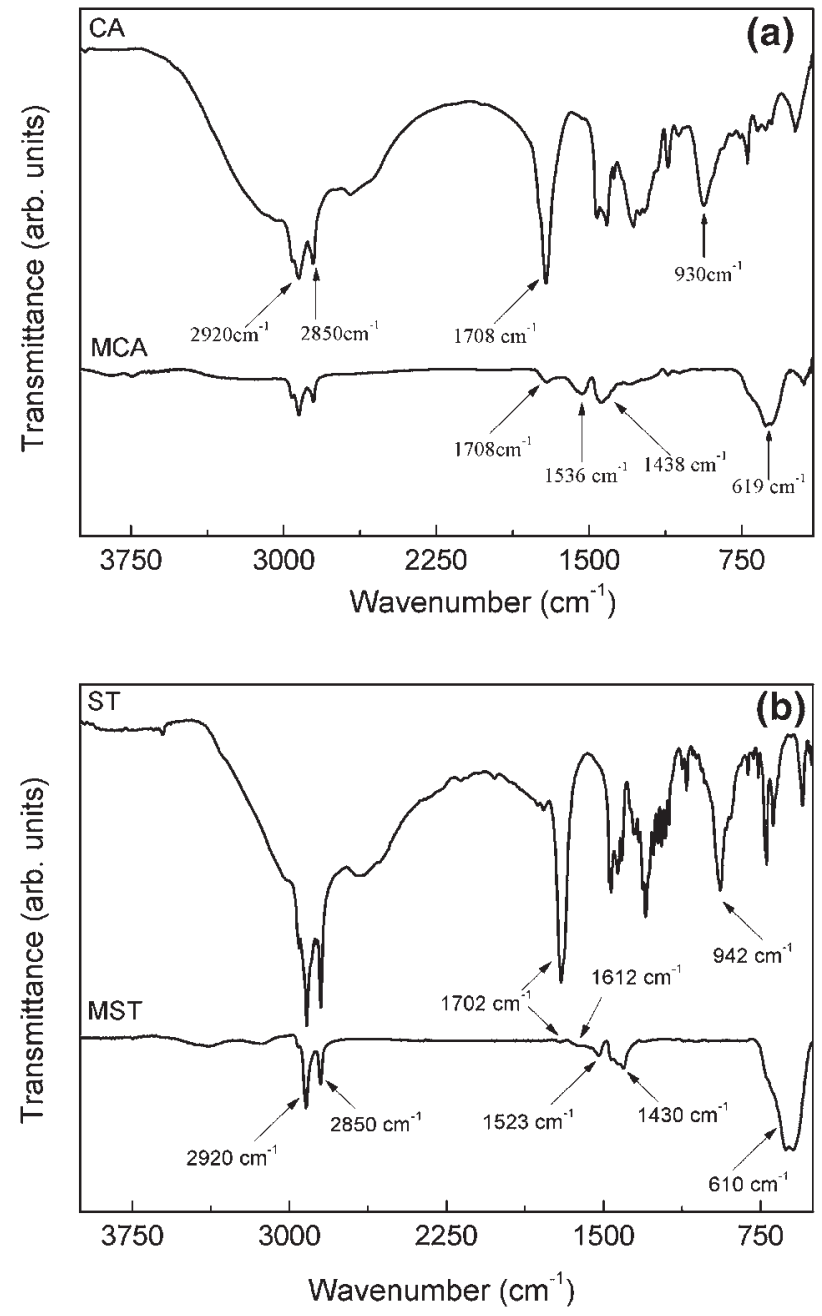

Figure 4. Infrared spectra of (a) capric-acid-coated sample (MCA) compared with that of neat capric acid (CA), and (b) stearic-acidcoated sample (MST) compared with that of neat stearic acid (ST).

surfactant in MCA [22]. This concluion is supported by the thermogravimetric analysis data, which also provided information for the presence of the secondary surfactant layer on the nanoparticles surfaces and relatively larger amount of the secondary surfactant in MCA. The absence of the characteristic band corresponding to dimeric carboxylic acids over $\sim 930-940 \mathrm{~cm}^{-1}$ indicates that the fatty acid molecules in the coated samples are in their monomeric form.

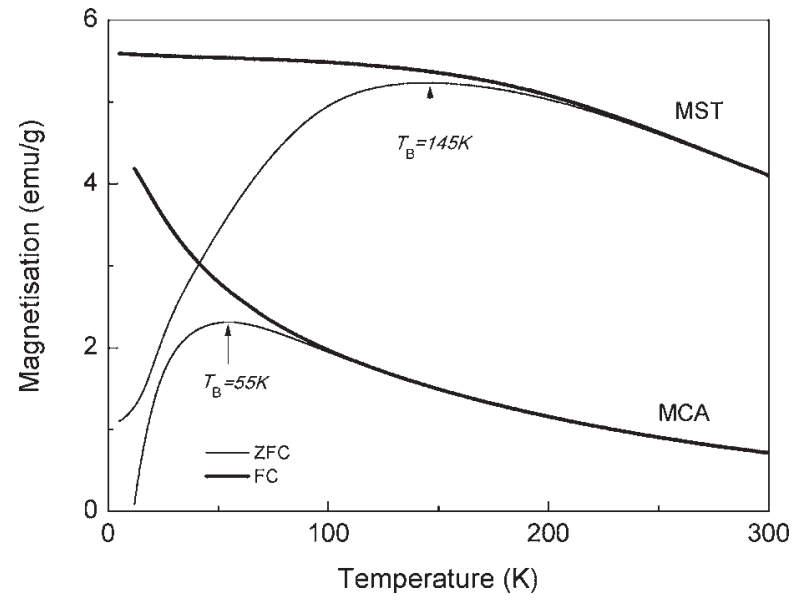

Figure 5. Temperature-dependent FC (thick line) and ZFC (thin line) magnetization curves of the capric acid (MCA) and stearic acid (MST)-coated powder samples.

Temperature-dependent magnetization studies have been carried out to get more information on the magnetic nature of the particles. The magnetizations of the samples measured under zero-field-cooled (ZFC) and field-cooled (FC) conditions, in a magnetic field of $50 \mathrm{Oe}$, are shown in figure 5. The nature of the FC and ZFC magnetization curves suggest superparamagnetic nature of the particles. A maximum is observed in the ZFC magnetization curve for both the coated samples and the temperature at which the magnetization of the sample reaches a maximum value is considered as the superparamagnetic blocking temperature $\left(T_{\mathrm{B}}\right)$. The observed blocking temperatures for MCA and MST are 55 and $145 \mathrm{~K}$, respectively (figure 5). The blocking temperature of the capric-acid-coated sample is much less compared with that for the stearic-acid-coated sample. This could be due to the presence of relatively larger particles in the stearic-acid-coated sample $(9.1 \mathrm{~nm})$ compared to that in the capric acid $(7.4 \mathrm{~nm})$ coated sample [32]. Similarly, the broad ZFC curve of the stearic-acid-coated sample is due to the wider particle size distribution [21]. The difference in the blocking temperature could also be due to the difference in the anisotropy contribution from inter-particle interaction. The presence of the larger clusters in the stearic-acid-coated sample can contribute more to the anisotropy energy, and the larger inter-particle interaction leads to increase in the blocking temperature as compared 


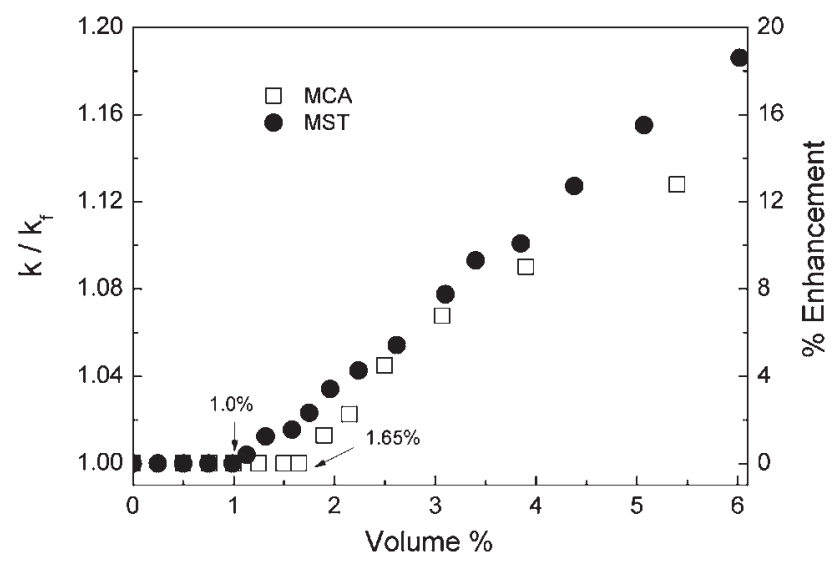

Figure 6. Variation of the thermal conductivity ratio with the concentration of the particles for capric acid (MCA) and stearic acid (MST)-coated nanofluids.

with the well-separated capric-acid-coated sample (as shown in the TEM image) [33,34].

The nature of the FC magnetization curve below the blocking temperature can give information on the interparticle interactions. A continuous decrease in the magnetization with increasing temperature indicates that the particles are non-interacting, whereas a flat curve with saturation trend in the magnetization indicates large inter-particle interactions. The continuous decrease in the magnetization with temperature indicates that the inter-particle interaction in the capric-acid-coated sample is suppressed and the particles are well separated from one another [33,34]. However, the slight flatness in the FC curve of the stearic-acid-coated sample, at low temperatures, indicates weak inter-particle interactions, probably due to the slight aggregation/assembly of the nanoparticles. The TEM studies also support the results from temperature-dependent magnetization measurements, where the capric-acid-coated sample is found to form well-separated particles, whereas the stearic-acid-coated sample shows assembly of particles forming aggregates (figure 2).

\subsection{Thermal conductivity of fluids}

Thermal conductivity of the nanofluids obtained by dispersing the nanoparticles of MCA and MST in toluene at different concentrations (volume\%) is measured. Variation of the thermal conductivity ratio $k / k_{\mathrm{f}}$, where $k$ and $k_{\mathrm{f}}$ are the thermal conductivities of the nanofluid and the base fluid (toluene), respectively, with the concentration of the particles is shown in figure 6. For both fluids, there is no change in the thermal conductivity from that of the base fluid, up to a certain concentration. With further increase in the concentration of the nanoparticles, thermal conductivity is increased almost linearly with the concentration of the particles. A similar trend in the thermal conductivity enhancement with the concentration of the nanoparticles has been reported for different fatty-acid-coated magnetite nanoparticles dispersed in different base fluids $[22,33,35,36]$.

The concentration above which the thermal conductivity of the magnetite nanofluid starts to increase over that of the base fluid is the critical concentration of that fluid for the thermal conductivity enhancement. The critical concentration is observed as $1.65 \mathrm{vol} \%$ for MCA and $1.0 \mathrm{vol} \%$ for MST, and the critical concentration is lower in the case of the stearicacid-coated fluid compared with the capric-acid-coated fluid. The unchanged thermal conductivity with the volume $\%$ of the nanoparticles, at lower concentrations, is probably due to the fact that the particles are well separated from each other and therefore, the thermal conductivity is only due to the conduction through the base fluid. It is also possible that the enhancement is much smaller at the concentrations below the critical concentration, which can be within the sensitivity limit of the measurement set-up. With the concentration increasing above the critical concentration, the particles come closer to each other and can form small clusters in the fluid through weak van der Waals and magnetic interactions. The number of these clusters increases on increasing the concentration of the particles in the nanofluid and leads to increase in the thermal conductivity. The deviation from linearity in the enhancement in the thermal conductivity at higher concentration for the capric-acid-coated sample could be due to the formation of larger clusters, which could create less concentrated liquid matrix in the nanofluid, because of the larger separation between the clusters. Even though the clusters have high thermal conductivity, the liquid matrix with less concentration and low thermal conductivity slightly reduces the thermal conductivity at higher concentrations. Moreover, there is a possibility for sedimentation of the larger clusters in the fluid due to the gravitational force. The stabilization ability of the 10-carbon capric acid is less effective as compared with the 18-carbon stearic acid to suspend the larger clusters, as reported [13]. It is reported that short-chain fatty acids can stabilize only smaller particles, whereas long-chain stearic acid stabilizes particles with a wider range of diameters [13].

The enhancement in the thermal conductivity at a lower concentration for the fluid of stearic-acid-coated sample could be due to the formation of clusters at low concentrations. The cluster formation at lower concentrations for the stearic-acidcoated sample is due to the larger hydrophobic interaction between the long chain (18-carbon) fatty acid compared with the short chain (10-carbon) fatty acid. Unlike the fluid of the capric-acid-coated sample, the fluid of MST shows linear enhancement in the thermal conductivity even at higher concentrations. This indicates that the saturated long chain fatty acid stabilizes the clusters even at higher concentrations and linear enhancement in the thermal conductivity is observed with increasing concentration of the nanoparticles in the fluid. Thermal conductivity at a particular concentration (4 vol\%) shows larger enhancement for the stearic-acid-coated fluid than for the capric-acid-coated fluid as shown in table 1.

Since the nanoparticles are magnetic, thermal conductivity of the two fluids in the presence of a DC magnetic field 


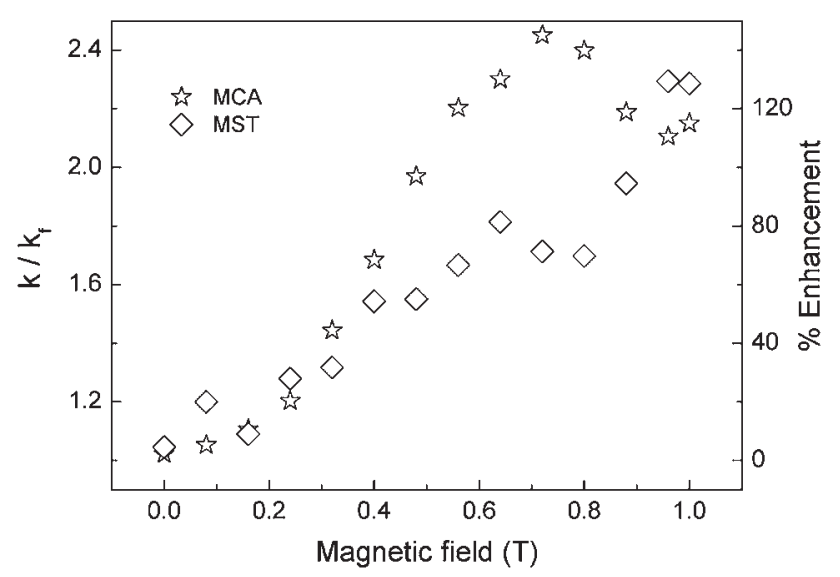

Figure 7. Comparison of the thermal conductivity enhancement of capric acid (MCA) and stearic acid (MST)-coated fluids (at $2.2 \mathrm{vol} \%$ ) as a function of magnetic field.

has been measured. Figure 7 shows a comparison of the thermal conductivity enhancement for the capric-acid- and stearic-acid-coated magnetite fluids in the presence of an applied magnetic field. Comparative studies are made at a concentration of $2.2 \mathrm{vol} \%$, which is larger than the critical concentrations of the stearic acid (1.0 vol\%) and capric acid (1.65 vol\%)-coated fluids. The thermal conductivity enhancement for the capric-acid-coated fluid is initially low in the low field region (up to $0.24 \mathrm{~T}$ ), increases at higher fields, reaches a maximum at $\sim 0.7 \mathrm{~T}$ and then decreases with further increase of magnetic field. However, in the case of the stearic-acidcoated fluid sample, the thermal conductivity increases almost linearly with increasing magnetic field up to the highest field. The observed enhancement is almost comparable for the fluids of MCA and MST up to a field of $0.4 \mathrm{~T}$. At higher fields (above $0.4 \mathrm{~T}$ ), the stearic-acid-coated fluid shows lesser enhancement in the thermal conductivity than that for the capric-acid-coated fluid. It is possible that the short-chain fatty acid (capric acid)-coated nanoparticles in the fluid show easy response to the applied magnetic field, due to the lower inter-particle magnetic interactions, and therefore show larger enhancement in the thermal conductivity. In the case of the long-chain fatty acid (stearic acid)-coated nanoparticles, slow response to the applied magnetic field leads to quantitatively less enhancement in the thermal conductivity. Moreover, the stearic-acid-coated nanoparticles could form loose clusters through inter-digitation of the surfactant molecules due to the larger hydrophobic interaction between the surfactants. The co-operative effect of the nanoparticles present in the loose clusters affects the response of nanoparticles to the applied magnetic field.

Thermogravimetric analysis showed that the amount of the surfactant present on the surface of the nanoparticles is less for the stearic-acid-coated magnetite nanoparticles (18\%) compared with the capric-acid-coated magnetite nanoparticles $(26 \%)$. Even though the amount of primary surfactant is comparable, the amount of the secondary surfactant is found to be double the amount on the capric-acid-coated nanoparticles. The difference in the amount of the secondary surfactant on the surface of the nanoparticles could also affect the response to the applied magnetic field. The number of secondary surfactant molecules on the surface of the nanoparticles can affect the dispersibility and the surfactant-solvent interaction, which affects the inter-particle dipolar interaction and hence the response of the nanoparticles in the presence of a magnetic field [22]. The increase in the thermal conductivity in the presence of a magnetic field is due to the increase in the dipolar interaction between the particles [36]. The dipolar interaction energy between the particles overcomes the thermal energy and the dipolar energy increases with increasing magnetic field [37]. The dipolar interaction energy aligns the nanoparticles in the direction of the applied field and forms chains of nanoparticles or dipolar chains in the fluid [36]. It is reported that the aspect ratio of the chain increases with increasing strength of the magnetic field and the heat energy is transferred through the back bone of the nanoparticle chains $[38,39]$.

Thus, the surfactant molecules attached on the surface of the nanoparticles play a crucial role in the clustering/aggregation of the nanoparticles in a fluid. Since the dispersed nanoparticles in the fluid are superparamagnetic in nature, the van der Waals interaction between the surfactants or between the particles contributes more to the aggregation of the nanoparticles as compared with the magnetic dipole-dipole interaction [40]. The surfactant molecule with the long aliphatic chain (stearic acid) attached to the nanoparticle's surface experiences more hydrophobic interaction with surfactants attached to the neighbouring particles. The strong hydrophobic interaction between the surfactants on the surface of the neighbouring particles leads to clustering of nanoparticles in the fluid through the inter-digitation of the surfactant molecules [33]. The long aliphatic chain of stearic acid may stabilize small clusters in the fluid, which hinders the formation of dipolar chains in a magnetic field, whereas the individual capric-acid-coated particles form chains easily and respond to the magnetic field quickly, giving rise to larger enhancement in the thermal conductivity in a magnetic field.

\section{Conclusions}

Magnetite nanoparticles coated with 10-carbon short-chain (capric acid) and 18-carbon long-chain (stearic acid) fatty acids are synthesized by a co-precipitation method. The amount of the surfactants and the nature of the attachment of the surfactant molecules on the surface of the nanoparticles were confirmed by TGA and IR studies. TEM images of the capric-acid-coated sample showed wellseparated particles and stearic-acid-coated sample showed formation of nanoparticle clusters. Magnetic measurements indicated the formation of superparamagnetic nanoparticles in both cases. The different nature of the FC magnetization curves indicated non-interacting nature of the 
particles (well separated) in capric-acid-coated sample and interacting nature of the particles (clusters) in stearic-acidcoated sample. Thermal conductivity measurements of the nanofluids in toluene showed a different critical concentration, below which there is no change in the thermal conductivity from that of the base fluid. The lower critical concentration for the stearic-acid-coated fluid could be due to the formation of clusters in the fluid at lower concentrations. Large enhancement in the thermal conductivity is obtained in a magnetic field for both fluids. The relatively larger enhancement in the thermal conductivity for the capric-acid-coated fluid than for the stearic-acid-coated fluid at higher magnetic fields could be due to easy response to the applied magnetic field of nanoparticles coated with the short-chain capric acid. The difference in the thickness of the surfactant layer due to the different chain lengths of the surfactants could be the reason for the difference in the enhancement in the thermal conductivity in a magnetic fluid.

\section{Acknowledgement}

R Lenin and A Dadwal are thankful to University Grants Commission (UGC) for financial support in the form of research fellowship.

\section{References}

[1] Saterlie M S, Sahin H, Kavlicoglu B, Liu Y M and Graeve O A 2012 Chem. Mater. 243299

[2] Murshed S M S, de Castro C A N and Lourenço M J V 2012 J. Nanofluids 1175

[3] Sun S and Zeng H 2002 J. Am. Chem. Soc. 1248204

[4] Park J, An K, Hwang Y, Park J G, Noh H J, Kim J Y et al 2004 Nat. Mater. 3891

[5] Tombácz E, Bica D, Hajdú A, Illés E, Majzik A and Vékás L 2008 J. Phys.: Condens. Matter 20204103

[6] Shen L F, Laibinis P E and Hatton T A 1999 Langmuir 15447

[7] Shen L F, Laibinis P E and Hatton T A 1999 J. Magn. Magn. Mater. 19437

[8] Guardia P, Batlle-Brugal B, Roca A G, Iglesias O, Morales M P, Serna C J et al 2007 J. Magn. Magn. Mater. 316 E756

[9] Filippousi M, Angelakeris M, Katsikini M, Paloura E, Efthimiopoulos I, Wang Y J et al 2004 J. Phys. Chem. C 118 16209

[10] Felicia L J, Johnson J C and Philip J 2014 J. Nanofluids 3328
[11] Yuan Y, Rende D, Altan C L, Bucak S, Ozisik R and Borca-Tasciuc D A 2012 Langmuir 2813051

[12] Younes H, Christensen G, Luan X N, Hong H P and Smith P 2012 J. Appl. Phys. 11164308

[13] Vékás L, Bica D and Marinica O 2006 Rom. Rep. Phys. 58257

[14] Fu L, Dravid V P and Johnson D L 2001 Appl. Surf. Sci. 181 173

[15] Barbeta V B, Jardim R F, Kiyohara P K, Effenberger F B and Rossi L M 2010 J. Appl. Phys. 1071

[16] Regmi R, Black C, Sudakar C, Keyes P H, Naik R, Lawes G et al 2009 J. Appl. Phys. 106113902

[17] Vekas L, Bica D, Gheorghe D, Potencz I and Rasa M 1999 J. Magn. Magn. Mater. 201159

[18] Avdeev M V, Bica D, Vekas L, Aksenov V L, Feoktystov A V, Marinica O et al 2009 J. Colloid Interface Sci. 33437

[19] Avdeev M V, Bica D, Vekas L, Marinica O, Balasoiu M, Aksenov V L et al 2007 J. Magn. Magn. Mater. 3116

[20] Patel H E, Das S K, Sundararajan T, Sreekumaran Nair A, George B and Pradeep T 2003 Appl. Phys. Lett. 832931

[21] Lenin R and Joy P A 2015 J. Nanofluids 4310

[22] Lenin R and Joy P A 2016 J. Phys. Chem. C 12011640

[23] Kuwahara Y, Zhang G, Wu J, Akai-Kasaya M, Saito A and Aono M 2002 Molecules 24

[24] Adschiri T, Takami S, Arita T, Hojo D, Minami K, Aoki N et al 2013 in Handbook of advanced ceramics: materials, applications, processing and properties S Somiya (ed) (Waltham, USA: Academic Press) p 949

[25] Adschiri T and Byrappa K 2009 in Nanohybridization of organic-inorganic materials A Muramatsu and T Miyashita (eds) (Heidelberg, Berlin: Springer-Verlag) p 247

[26] Lane C A, Burton D E and Crabb C C 1984 J. Chem. Educ. 61 815

[27] Zhao S Y, Lee D K, Kim C W, Cha R G, Kim Y H and Kang Y S 2006 Bull. Korean Chem. Soc. 27237

[28] Waldron R D 1955 Phys. Rev. 991727

[29] Bronstein L M, Huang X L, Retrum J, Schmucker A, Pink M, Stein B D et al 2007 Chem. Mater. 193624

[30] Ding X, Bao L, Jiang J and Gu H 2014 RSC Adv. 49314

[31] Yang K, Peng H, Wen Y and Li N 2010 Appl. Surf. Sci. 256 3093

[32] Liu C and Zhang Z J 2001 Chem. Mater. 132092

[33] Lenin R and Joy P A 2017 J. Colloid Interface Sci. 506162

[34] Vargas J, Nunes W, Socolovsky L, Knobel M and Zanchet D 2005 Phys. Rev. B. 72184428

[35] Lenin R and Joy P A 2017 Colloids Surf. A 529922

[36] Philip J, Shima P D and Raj B 2007 Appl. Phys. Lett. 91203108

[37] Mendelev V S and Ivanov A O 2004 Phys. Rev. E 70051502

[38] Philip J, Shima P D and Raj B 2008 Nanotechnology 19305706

[39] Shima P D, Philip J and Raj B 2009 Appl. Phys. Lett. 94223101

[40] Lalatonne Y, Richardi J and Pileni M P 2004 Nat. Mater. 3121 